\title{
Visualization of Protein Interactions in Living Cells Using Bimolecular Fluorescence Complementation (BiFC) Analysis
}

Protein interactions control many cellular functions. The functional characteristics of many proteins are determined by the proteins that they interact with in each cell. Interactions with different partners and in response to different extracellular signals can occur in different subcellular locations. Determination of the locations of protein complexes and their regulation can provide insight into the functional roles of the protein interactions.

This unit describes the bimolecular fluorescence complementation (BiFC) assay for the visualization of protein interactions in living cells (Hu et al., 2002). This approach is based on complementation between nonfluorescent fragments of the yellow fluorescent protein (YFP) when they are brought together by an interaction between proteins fused to each fragment (Fig. 19.10.1). The BiFC assay allows direct visualization of the subcellular locations of protein interactions in living cells (see Basic Protocol). This approach can be used for the analysis of many protein interactions and does not require information about the structures of the interaction partners.

Most proteins have a large number of alternative interaction partners in each cell. Many of these interactions are mutually exclusive, resulting in competition for shared interaction partners in the cell. To study the competition for interactions between alternative interaction partners in living cells, the authors of this unit have developed a multicolor BiFC assay by fusing fragments of different fluorescent proteins to the interaction partners (see Alternate Protocol 1). The multicolor BiFC assay is based on the use of fragments of fluorescent proteins with distinct spectral characteristics. Bimolecular complexes formed between these fragments can be visualized using different excitation and emission wavelengths, enabling parallel visualization of multiple interactions in the same cell. The

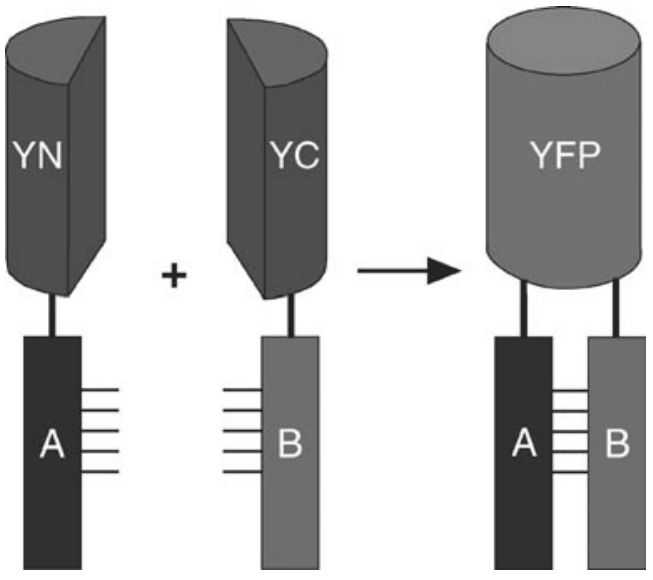

Figure 19.10.1 Schematic representation of the principle of the BiFC assay Two fragments (YN and $\mathrm{YC}$ ) of the yellow fluorescent protein (YFP) are fused to putative interaction partners (A and B). The association of the interaction partners allows formation of a bimolecular fluorescent complex. This black-and-white facsimile of the figure is intended only as a placeholder; for a full-color version of the figure go to http://www.interscience.wiley.com/c_p/colorfigures.htm. 
multicolor BiFC assay allows comparison of the subcellular locations of different protein interactions in the same cell, as well as analysis of the competition among mutually exclusive interaction partners for complex formation with a common partner (Alternate Protocol 2).

NOTE: Cells are cultured in a humidified $37^{\circ} \mathrm{C}, 5 \% \mathrm{CO}_{2}$ incubator. Cells can be incubated in a $30^{\circ} \mathrm{C}, 5 \% \mathrm{CO}_{2}$ incubator to promote maturation of the fluorophore and thereby increase the signal (see Commentary). Cells are maintained under conditions required for each line.

BASIC PROTOCOL
Bimolecular Fluorescence Complementation

\section{VISUALIZATION OF PROTEIN INTERACTIONS IN LIVING CELLS}

The BiFC assay allows direct visualization of protein interactions in living cells based on complementation between nonfluorescent fragments of YFP or other fluorescent proteins.

\section{Materials}

Plasmid vectors for expression of proteins of interest (also see Critical Parameter): e.g., pFlag-CMV2 (Sigma) or pHA-CMV (Clontech)

DNA encoding amino acid residues 1 to 154 of yellow fluorescent protein (YN fragment; also see Critical Parameters and Table 19.10.1)

DNA encoding amino acid residues 155 to 238 of yellow fluorescent protein (YC fragment; also see Critical Parameters and Table 19.10.1)

Linkers (see Critical Parameters)

DNA encoding proteins (interaction partners, wild-type) of interest

DNA encoding mutated, single amino acid-substitution variants of the protein of interest that do not interact with each other (controls)

Cells that can be transfected using plasmid DNA (preferably adherent, monolayer cell line)

Appropriate culture medium

FuGENE 6 (Roche Diagnostics) or other transfection reagent

Tissue culture vessels appropriate for experiment: e.g., cluster plates, slide chambers, or glass coverslips

Inverted fluorescence microscope equipped with:

Sensitive CCD camera

$20 \times$ to $100 \times$ objectives

Filters for visualization of YFP (excitation $500 \pm 10 \mathrm{~nm}$; emission $535 \pm 15 \mathrm{~nm}$ ) Software for instrument control and data analysis

Additional reagents and equipment for expressing proteins in mammalian cells (UNIT 5.10), mammalian cell culture (APPENDIX 3C), and immunoblotting (UNIT 10.10)

Table 19.10.1 Selection of Fluorescent Protein Fragments for BiFC and Multicolor BiFC Analyses

\begin{tabular}{lll}
\hline Fluorescent protein fragments & Applications & Filters \\
\hline YN155+YC155 & BiFC analysis & YFP \\
YN173+YC173 & BiFC analysis & YFP \\
CN155+CC155 & BiFC analysis & CFP \\
YN155+CN155+CC155 & Multicolor BiFC analysis & CFP \\
& & YFP \\
\hline
\end{tabular}

\subsection{2}




\section{Construct plasmid vectors}

1. Using the appropriate plasmid vectors, construct expression vectors by fusing the sequences encoding amino acid residues 1 to 154 (YN) and residues 155 to 238 (YC) of the yellow fluorescent protein to sequences encoding the proteins of interest. Whenever possible, test fusions to both the $\mathrm{N}$ - and $\mathrm{C}$-terminal ends of the proteins to be investigated. As controls, construct plasmids encoding mutated variants of the proteins that do not interact with each other.

For additional information about the design of fusion constructs, see Critical Parameters. The chimeric coding regions can be expressed in mammalian cells using vectors such as pFlag-CMV2 (Sigma) or pHA-CMV (Clontech).

As an alternative, fragments of the cyan fluorescent protein (CFP) may be used (see Table 19.10.1).

\section{Prepare cells}

2. Seed cells the day before transfection at a density that will allow for cell proliferation over the course of the experiment and, at the same time, allow for the effects of cell growth and density on the interaction under investigation.

The BiFC assay can be used for the analysis of protein interactions in many mammalian cell lines. The authors have studied protein interactions using the BiFC assay in COS-1, HEK293, HeLa, Hep3B, $\alpha$ TN4, and NIH3T3 cells (Hu et al., 2002; Grinberg et al., 2004; Rajaram and Kerppola, 2004).

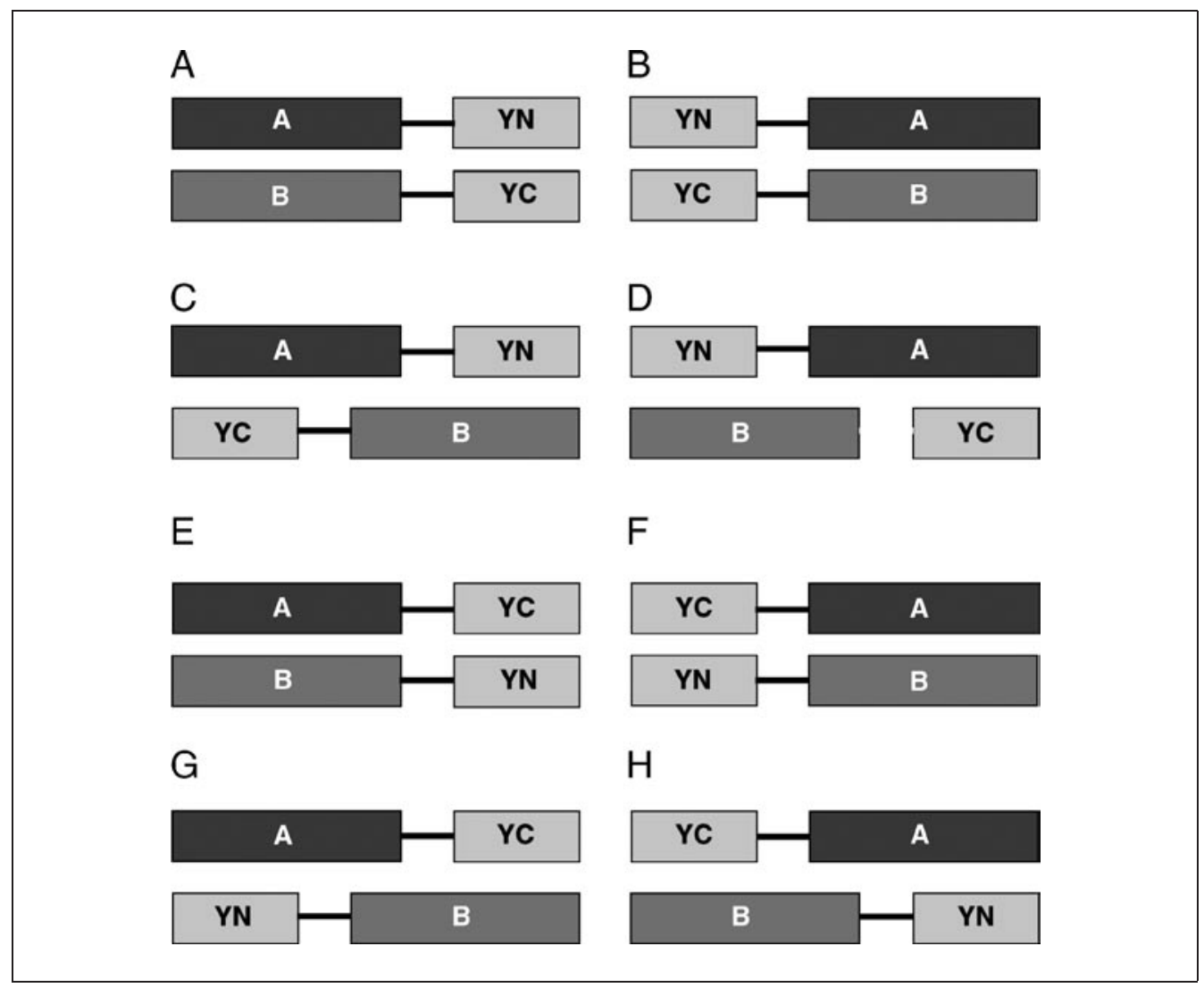

Figure 19.10.2 Multiple combinations of fusion proteins should be tested for bimolecular fluorescence complementation. Amino- and carboxyl-terminal fusions can be used to test eight distinct combinations $(\mathbf{A}$ to $\mathbf{H})$. This black-and-white facsimile of the figure is intended only as a placeholder;

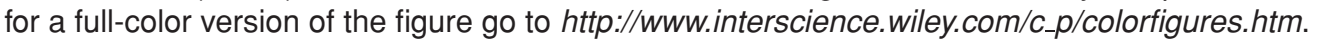

Identification of Protein Interactions

19.10 .3 
Cluster plates are convenient for processing multiple transfections in parallel. If shortworking-distance objectives will be used to visualize the interaction, the cells should be grown in slide chambers or on glass coverslips.

APPENDIX 3C describes basic mammalian cell culture techniques.

3. When cells reach appropriate confluence (e.g., $\sim 50 \%$ ), transfect cells with appropriate amounts (e.g., $0.25 \mu \mathrm{g}$ ) of the BiFC plasmid (from step 1) encoding the fusion proteins. As controls, transfect cells in parallel with the plasmids encoding mutated variants of the proteins that do not interact with each other.

FuGENE 6 from Roche Diagnostics is used according to the manufacturer's instructions to introduce plasmid DNA into cells, as it has low background fluorescence and requires minimal manipulation of the cells. Users need to follow specific protocols and optimize the conditions of transfection if other transfection reagents are used.

4. Grow cells until fluorescence is detected (12 to $36 \mathrm{hr}$ ). If necessary, incubate the cells at $30^{\circ} \mathrm{C}$ with a $5 \% \mathrm{CO}_{2}$ atmosphere to promote maturation of the fluorophore and to increase the signal (however, interpret results obtained under such conditions with care, as incubation at a lower temperature could alter protein interactions). Compare the levels of fusion protein expression with those of the endogenous proteins by immunoblot analysis (see UNIT 10.10).

\section{Examine cells}

5. Wash the cells with PBS to remove dead cells and debris, then add fresh medium.

6. View the cells using an inverted fluorescent microscope with a $20 \times$ long-workingdistance objective and excitation at $500 \pm 10 \mathrm{~nm}$ with emission detection at 535 $\pm 15 \mathrm{~nm}$. Confirm that fluorescent cells are alive and exhibit normal morphology compared to nontransfected cells.

Higher-numerical-aperture objectives may be necessary to visualize weak signals and higher magnification may be helpful to determine the subcellular localization.

Cells grown on coverslips can be fixed and individual proteins can be visualized by indirect immunofluorescence analysis.

If CFP is used as the reporter, the excitation wavelength is $436 \pm 5 \mathrm{~nm}$ and the emission wavelength is $470 \pm 15 \mathrm{~nm}$.

\section{Assess results}

7. Compare results obtained with cells transfected with the wild-type interaction proteins and those transfected with the control, mutant constructs with single amino acid substitutions that prevent interactions. Compare the number of fluorescent cells observed in cultures expressing fluorescent protein fragment fusions with that observed in cultures expressing intact fluorescent proteins.

Fluorescence in a small subpopulation of cells is difficult to interpret, since it may represent complementation due to nonspecific interactions, or it may reflect a regulated interaction that occurs only in response to signaling in a subset of cells.

The following conclusions may be drawn:

Specific interaction: If fluorescence is detected when wild-type proteins are expressed, and this signal is eliminated or significantly reduced by single amino acid substitutions that prevent the interaction, it is likely that the bimolecular fluorescence complementation represents a specific interaction between the proteins fused to the fragments of fluorescent proteins.

Bimolecular 
Nonspecific interaction: If mutations that are known to eliminate the interaction do not reduce or eliminate the fluorescence, then the bimolecular complementation is due to nonspecific interactions between the fusion proteins. If this is the case, the BiFC assay may not be an appropriate assay for the study of the two proteins. Alternatively, a different combination of fusion proteins or linkers should be tested.

No fluorescence complementation detected: The lack of fluorescence complementation in the BiFC assay does not prove the absence of an interaction, even if coexpression of the same fusion proteins with other interaction partners results in bimolecular fluorescence complementation. Factors that may contribute to this are discussed in the Commentary. Different combinations of BiFC constructs or alternative approaches should be employed.

\section{QUANTIFICATION OF FLUORESCENCE COMPLEMENTATION EFFICIENCY}

Quantification of the efficiency of fluorescence complementation is useful for comparison of interactions involving closely related proteins. It is particularly important for determining whether fluorescence complementation represents a specific interaction by comparison of wild-type and mutated proteins. The relative efficiencies of bimolecular fluorescence complementation by structurally unrelated proteins cannot be used to determine the efficiencies of complex formation, since many factors unrelated to the efficiency of complex formation influence the efficiency of bimolecular fluorescence complementation.

\section{Additional Materials (also see Basic Protocol)}

Plasmid encoding full-length CFP (assuming YFP fragments are used in the Basic Protocol) or other spectrally distinguishable fluorescent protein

Filters for visualization of YFP (excitation $500 \pm 10 \mathrm{~nm}$; emission $535 \pm 15 \mathrm{~nm}$ ) and CFP (excitation $436 \pm 5 \mathrm{~nm}$; emission $470 \pm 15 \mathrm{~nm}$ ) fluorescence

1. Follow steps 1 to 5 of the Basic Protocol, but add 50 ng of a plasmid encoding full-length CFP or another spectrally distinguishable fluorescent protein into each transfection.

2. View cells with a fluorescence microscope using filters optimized for separation of CFP and YFP signals. Collect images of at least 100 cells that show detectable fluorescence using both filter sets.

3. Quantify the fluorescence intensities using both YFP and CFP filters integrated over the area of each cell. Subtract the background in an area of the field containing no cells.

4. Calculate the ratio of YFP/CFP fluorescence for each cell.

This represents the complementation efficiency for the combination of proteins expressed in individual cells.

5. Plot the data in a histogram by binning cells with similar ratios as shown in Fig. 19.10.3.

6. Assess result.

If a mutation significantly reduces the YFP/CFP ratio compared to the wild-type protein (but the levels of expression and localization of the proteins are not altered), the observed fluorescence is likely to represent a specific interaction between the proteins. If the ratios for wild-type and mutated proteins are similar, the observed fluorescence signal may represent nonspecific fluorescence complementation, or the mutation may not affect the interaction between the proteins.
SUPPORT PROTOCOL 1

Identification

of Protein

Interactions

19.10 .5 


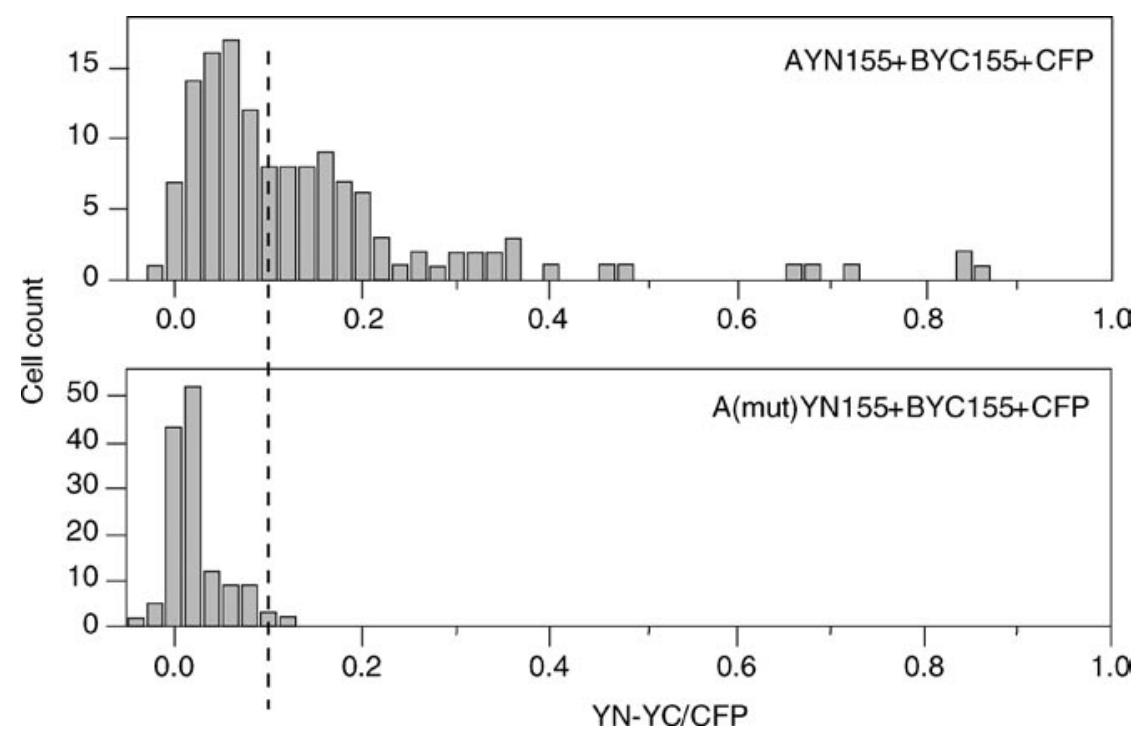

Figure 19.10.3 The effects of mutations that eliminate the interaction interface should be tested on the efficiency of bimolecular fluorescence complementation (data adapted from Hu et al., 2002). Plasmids encoding either wild-type (upper panel) or mutated (lower panel) interaction partners were transfected into cells together with an internal reference (CFP). The fluorescence intensities produced by bimolecular fluorescence complementation (YN-YC) and the internal reference (CFP) were measured in individual cells. The distribution of ratios between the fluorescence intensities in individual cells is plotted in each histogram. The dashed line indicates the maximal ratio produced by nonspecific interactions.

ALTERNATE PROTOCOL 1

Bimolecular Fluorescence Complementation

19.10.6

\section{SIMULTANEOUS VISUALIZATION OF MULTIPLE PROTEIN INTERACTIONS IN THE SAME CELL}

Comparison of the subcellular localization of different protein-protein complexes can provide information about similarities and differences in their functions based on colocalization or nonoverlapping distributions of the complexes. The locations of different complexes can be compared in different cells by using markers for various cellular structures to define the localization of each complex separately. However, it is often difficult to find markers that precisely match the localization of a particular complex. It is therefore preferable to compare the locations of protein complexes in the same cell. This protocol describes a multicolor BiFC approach that enables simultaneous visualization of several interactions in the same cell (Hu and Kerppola, 2003; Grinberg et al., 2004).

The multicolor BiFC assay is based on complementation between fragments of different fluorescent proteins that produce bimolecular fluorescent complexes with distinct spectra. These fragments are fused to alternative interaction partners such that complexes formed with different partners can be visualized independently in the same cell using different excitation and emission wavelengths. This approach provides a unique method for comparison of the distributions of different protein complexes in the same cell and for analysis of the relative efficiencies of interactions with mutually exclusive interaction partners (Alternate Protocol 2).

\section{Additional Materials (also see Basic Protocol)}

DNA encoding amino acid residues 155 to 238 of cyan fluorescent protein (also see Critical Parameters and Table 19.10.1)

DNA encoding several alternative interaction partners 
DNA encoding amino acid residues 1 to 154 of the cyan and yellow fluorescent proteins (also see Critical Parameters and Table 19.10.1)

Interference filters for fluorescence microscope designed to minimize cross-talk between the fluorescence signals from bimolecular fluorescent complexes formed by fragments of different fluorescent proteins (see below)

\section{Construct plasmid vectors}

1. Using the appropriate plasmid vectors, construct mammalian expression vectors by fusing the sequences encoding amino acid residues 155 to 238 of the cyan fluorescent protein $(\mathrm{CFP})$ to the protein whose interactions are to be investigated (this protein is designated Z-CC155), and fuse sequences encoding amino acid residues 1 to 154 of YFP and residues 1 to 154 of CFP to two alternative interaction partners (these proteins are designated A-YN155 and B-CN155, respectively). As controls, construct plasmids encoding these fluorescent protein fragments fused to the same interaction partners.

For additional information about the design of fusion constructs, see Critical Parameters.

Other fluorescent proteins whose fragments produce bimolecular fluorescent complexes with distinct spectra may be used (Hu and Kerppola, 2003; also see Critical Parameters).

\section{Prepare cells}

2. Seed cells the day before transfection at a density that will allow for cell proliferation over the course of the experiment and, at the same time, allow for the effects of cell growth and density on the interaction under investigation.

If short-working-distance objectives will be used to visualize the interaction, the cells should be grown in slide chambers or on glass coverslips.

3. When cells reach appropriate confluence (e.g., $\sim 50 \%$ ), transfect cells with an appropriate amounts (e.g., $0.25 \mu \mathrm{g}$ ) of the plasmids encoding A-YN155, B-CN155, and Z-CC155. As a control, transfect each pair of expression vectors (A-YN155 and $\mathrm{Z}-\mathrm{CC} 155$ as well as B-CN155 and Z-CC155) into separate cells.

4. Grow cells until fluorescence from both complexes (i.e., A-YN155-Z-CC155 and B-CN155-Z-CC155) is detected using filters optimized for the detection of YFP and CFP fluorescence, respectively. If necessary, incubate the cells at $30^{\circ} \mathrm{C}$ with $5 \%$ $\mathrm{CO}_{2}$ to promote maturation of the fluorophores and to increase the signal (however, interpret results obtained under such conditions with care, as incubation at a lower temperature could alter protein interactions).

5. Compare the levels of expression of the proteins by immunoblot analysis and adjust the amounts of plasmids transfected if necessary.

\section{Examine cells}

6. Wash the cells with PBS to remove dead cells and debris, then add fresh medium.

7. View the cells using an inverted fluorescence microscope with a $20 \times$ long-workingdistance objective.

To view the fluorescence emissions of A-YN155-Z-CC155 and B-CN155-Z-CC155 complexes use two filter sets with excitation at $500 \pm 10 \mathrm{~nm}$ and emission at $535 \pm 15 \mathrm{~nm}$ (to detect $Y N-C C$ complexes), and excitation at $436 \pm 5 \mathrm{~nm}$ and emission at $470 \pm 15 \mathrm{~nm}$ (to detect $\mathrm{CN}-\mathrm{CC}$ complexes). The emission and excitation wavelengths are separated using a dichroic mirror with transmission windows at 450 to $490 \mathrm{~nm}$ and 520 to $590 \mathrm{~nm}$.

The fluorescence emissions of YN155-CC155 and CN155-CC155 bimolecular fluorescent complexes can be resolved with less than $2 \%$ cross-talk between the signals using the filters optimized for YFP and CFP detection.

Identification 
ANALYSIS OF THE COMPETITION BETWEEN ALTERNATIVE INTERACTION PARTNERS IN LIVING CELLS

The selectivity of protein interactions is determined by the relative binding affinities of alternative interaction partners and by the local concentrations of each protein. It is difficult to predict the selectivity of protein interaction in cells based on in vitro studies, since many factors, including covalent modifications, differences in subcellular distributions, interactions with additional proteins, and binding affinity, can influence the selectivity of interactions. The following protocol describes a quantitative multicolor BiFC assay that can provide information about the relative efficiencies of complex formation by mutually exclusive interaction partners in living cells (Hu and Kerppola, 2003; Grinberg et al., 2004).

\section{Principles of competition experiment}

To investigate the competition between two alternative interaction partners (i.e., A and B) for a shared partner (i.e., Z), the proteins are fused to fragments of different fluorescent proteins that can form spectrally distinct bimolecular fluorescent complexes (i.e., AYN155, B-CN155, and Z-CC155). Equal concentrations of the two competing interaction partners (i.e., A-YN155 and B-CN155) are expressed with a limiting concentration of the shared partner (i.e., Z-CC155). The fluorescence intensities of bimolecular fluorescent complexes formed by the alternative interaction partners (i.e., A-YN155-Z-CC155 and B-CN155-Z-CC155) are measured in the same cells. For purposes of this protocol, the phrase "fluorescence intensities of complexes" and variations thereof will be used to refer to the fluorescence intensities of individual cells with specific spectral characteristics.

\section{Additional Materials (also see Basic Protocol)}

Constructs prepared in Alternate Protocol 1, step 1

\section{Prepare cells}

1. Seed cells the day before transfection at a density that will allow for cell proliferation over the course of the experiment and, at the same time, allow for the effects of cell growth and density on the interaction under investigation.

2. When cells reach appropriate confluence (e.g., $\sim 50 \%$ ), transfect cells with an appropriate amount (e.g., $0.05 \mu \mathrm{g}$ ) of the plasmid encoding Z-CC155 to produce a limiting concentration of the protein (established by immunoblot analysis) and appropriate amounts (i.e., $0.25 \mu \mathrm{g}$ ) of the plasmids encoding A-YN155 and B-CN155 to produce equal amounts of these proteins that are in molar excess relative to Z-CC155. For quantitation using absolute fluorescence intensities (see Support Protocol 2), also transfect each pair of expression vectors (A-YN155 and Z-CC155; as well as B-CN155 and Z-CC155) in separate cells. For quantitation using relative fluorescence intensities (see Support Protocol 3), also transfect expression vectors encoding fusions with the interaction partners fused to different combinations of fluorescent protein fragments (i.e. A-YN155 + A-CN155 + Z-CC155; as well as B-YN155 + A-CN155 + Z-CC155) into separate cells.

3. Grow cells until fluorescence from both complexes (i.e., A-YN155-Z-CC155 and $\mathrm{B}-\mathrm{CN} 155-\mathrm{Z}-\mathrm{CC} 155)$ is detected. If necessary, incubate the cells at $30^{\circ} \mathrm{C}$ with a $5 \%$ $\mathrm{CO}_{2}$ atmosphere to promote maturation of the fluorophore and to increase the signal (however, interpret results obtained under such conditions with care, as incubation at a lower temperature could alter protein interactions).

Bimolecular Fluorescence Complementation
4. Compare the levels of expression of the proteins and adjust the amounts of plasmids transfected if necessary. 


\section{Examine cells}

5. Wash the cells with PBS to remove dead cells and debris and add fresh medium.

6. View the cells using an inverted fluorescence microscope with a $20 \times$ long working distance objective. Identify cells that are healthy and that have a morphology similar to nontransfected cells.

Interactions between competing partners will result in the formation of two BiFC complexes, A-YN155-Z-CC155 and B-CN155-Z-CC155, with distinct spectral characteristics. To image the fluorescence emissions of A-YN155-Z-CC155 and B-CN155-Z-CC155 complexes, use filters with excitation at $500 \pm 10 \mathrm{~nm}$ and emission at $535 \pm 15 \mathrm{~nm}$ to detect YN-CC complexes and filters with excitation at $436 \pm 5 \mathrm{~nm}$ and emission at $470 \pm 15 \mathrm{~nm}$ to detect $\mathrm{CN}-\mathrm{CC}$ complexes. The emission and excitation light are separated using a dichroic mirror with transmission windows at 450 to $490 \mathrm{~nm}$ and 520 to $590 \mathrm{~nm}$.

\section{QUANTITATION OF MULTICOLOR BiFC ANALYSIS}

The authors of this unit have developed two methods for quantification of data from the multicolor BiFC assay that can provide information about the relative efficiencies of complex formation by mutually exclusive interaction partners in living cells ( $\mathrm{Hu}$ and Kerppola, 2003; Grinberg et al., 2004). The first method, designated absolute competition (Support Protocol 2), is based on comparison of the absolute fluorescence intensities of bimolecular fluorescent complexes produced when a pair of interaction partners is expressed in the presence and absence of an alternative interaction partner in separate cells. The second method, designated relative competition (Support Protocol 3), is based on comparison of the relative fluorescence intensities of bimolecular fluorescent complexes produced when a protein is expressed together with two alternative interaction partners in the same cells. It is best to use both methods in combination to eliminate potential caveats inherent in each approach.

\section{Comparison of the Absolute Fluorescence Intensities of Bimolecular Fluorescent Complexes Produced When a Pair of Interaction Partners Is Expressed in the Presence and Absence of a Competitor in Separate Cells}

The absolute fluorescence intensities of bimolecular fluorescent complexes are compared when two fusion proteins are expressed alone (i.e. A-YN155+Z-CC155) and when they are expressed in the presence of a competitor (i.e. A-YN155+B-CN155+Z-CC155) in separate cells. This approach is designated absolute competition. This method is well suited for analysis of large differences in the efficiencies of complex formation between alternative interaction partners. Since it involves comparison of different cell populations, it is subject to error due to variations between the populations. Since the absolute fluorescence intensities vary between individual cells, this requires comparison of the fluorescence intensities of the populations or representative subsets of the populations. Using this approach, it is generally possible to detect only large differences in the efficiencies of complex formation. This approach compares the fluorescence intensities of bimolecular fluorescent complexes formed by the same interacting pair, in the absence and presence of competitor. This circumvents the effects of differences in the steric arrangement of the fluorescent protein fragments between different interaction partners that prevent comparison of the efficiencies of complex formation based on the absolute fluorescence intensities of different bimolecular fluorescent complexes. 
Carry out Alternate Protocol 2, steps 1 to 6, then continue with the following steps.

1. Measure the fluorescence intensities of YN-CC complexes in 100 to 300 cells expressing A-YN155 + Z-CC155.

2. Measure the fluorescence intensities of YN-CC complexes in 100 to 300 cells expressing A-YN155 + B-CN155 + Z-CC155.

3. Measure the fluorescence intensities of CN-CC complexes in 100 to 300 cells expressing B-CN155 + Z-CC155.

4. Measure the fluorescence intensities of CN-CC complexes in 100 to 300 cells expressing A-YN155 + B-CN155 + Z-CC155.

5. Compare the fluorescence intensities of $\mathrm{YN}-\mathrm{CC}$ complexes measured in steps 1 and 2 as well as the $\mathrm{CN}-\mathrm{CC}$ complexes measured in steps 3 and 4.

6. Assess results.

Case i: If the fluorescence intensities of the two bimolecular fluorescent complexes (i.e., A-YN155-Z-CC155 and B-CN155-Z-CC155) expressed in the same cells are $\sim 50 \%$ of the fluorescence intensities of the complexes when expressed separately, then the two complexes form with comparable efficiencies.

Case ii: If the fluorescence intensity of one complex is similar whether it is expressed separately or in combination, whereas the fluorescence intensity of the other complex is reduced by $>50 \%$ when expressed in combination, then the former complex forms with higher efficiency than the latter.

Case iii: If the fluorescence intensities of both complexes expressed together are equal or higher than those observed when they are expressed separately, then the interaction partners do not compete for complex formation. The shared interaction partner may not be present at limiting concentration, and the relative efficiencies of complex formation cannot be determined based on the data.

Case iv: If the fluorescence intensities of both complexes are reduced by significantly more than 50\% when they are expressed together, or if the changes in fluorescence intensities are not reciprocal, then the interaction partners may affect the expression or functions of each other, and the relative efficiencies of complex formation cannot be determined based on the data.

SUPPORT PROTOCOL 3

Bimolecular Fluorescence Complementation

\section{Comparison of the Relative Fluorescence Intensities of Bimolecular Fluorescent Complexes Produced when a Protein Is Expressed with Two Alternative Interaction Partners in the Same Cells}

The relative fluorescence intensities of bimolecular fluorescent complexes are compared when a fusion protein is expressed with two alternative interaction partners fused to fragments of different fluorescent proteins (i.e., A-YN155 + B-CN155 + Z-CC155). This approach is designated relative competition. This approach is suitable for detection of relatively modest differences in the efficiencies of complex formation between structurally related alternative interaction partners. Since this approach compares complexes in the same cells, it is less sensitive to variations between cells. However, since this approach compares the relative fluorescence intensitites of bimolecular fluorescent complexes produced by different interaction partners, it is subject to variations in the efficiency of association of the fluorescent protein fragments when they are fused to different interaction partners. Therefore, this approach can be used only when there are no large differences in the steric constraints to association of the fluorescent protein fragments between different complexes.

\subsubsection{0}


Carry out Alternate Protocol 2, steps 1 to 6, then continue with the following steps.

1. Measure the fluorescence intensities of 100 to 300 cells expressing A-YN155 + $\mathrm{A}-\mathrm{CN} 155+\mathrm{Z}-\mathrm{CC} 155$ to use as a reference.

2. Measure the fluorescence intensities of 100 to 300 cells expressing A-YN155 + $\mathrm{B}-\mathrm{CN} 155+\mathrm{Z}-\mathrm{CC} 155$.

3. Measure the fluorescence intensities of 100 to 300 cells expressing B-YN155 + A-CN155 + Z-CC155.

4. Plot the fluorescence intensities of $\mathrm{YN}-\mathrm{CC}$ versus $\mathrm{CN}-\mathrm{CC}$ complexes for each individual cell as a scatter plot as shown in Figure 19.10.4 (middle row).

5. Compare the slopes produced by different combinations of interaction partners. The relative slopes of these plots provide information about the relative efficiencies of complex formation between alternative interaction partners. Alternatively, calculate the ratio $\mathrm{YN}-\mathrm{CC} /(\mathrm{YN}-\mathrm{CC}+\mathrm{CN}-\mathrm{CC})$ and plot the data as a histogram as shown in Figure 19.10.4 (bottom row). The distribution of ratios in the population can provide information about the relative efficiencies of complex formation.

6. Assess results.

Case $i$ : If the relative fluorescence intensities of all combinations of complexes display a linear relationship, and the slopes of the relationships are not significantly different, then the two interaction partners compete with indistinguishable efficiencies for the shared partner.

Case ii: If the fluorescence intensities of all combinations of complexes display a linear relationship, and the slopes of the relationships are significantly different, then the two interaction partners compete with different efficiencies for the shared partner.

Case iii: If the fluorescence intensities of one or all combinations of complexes do not display a linear relationship, or if the slopes cannot be determined with sufficient confidence, then the relative efficiencies of competition cannot be determined based on the data.

\section{Comparison of Results from the Absolute and Relative Competition Approaches}

The relative competition approach (Support Protocol 3) is generally more accurate for complexes with similar structures. However, this approach can only be used when there are no differences in the steric constraints to association of the fluorescent protein fragments.

To confirm that the identities of the fluorescent protein fragments fused to the alternative interaction partners do not influence the relative efficiencies of complex formation, it is necessary to exchange the fragments between the interaction partners (i.e., compare A-YN155 + B-CN155 + Z-CC155 with B-YN155 + A-CN155 + Z-CC155). In the absolute competition approach, exchange of the fragments should not affect the difference in fluorescence intensity in the presence and absence of the competitor. In the relative competition approach, the slope of the plot of YN-CC versus $\mathrm{CN}-\mathrm{CC}$ intensities should shift in the opposite direction relative to the slope observed for the reference complexes (A-YN155 + A-CN155 + Z-CC155; compare the slopes of the plots on the right and left of the middle row of Fig. 19.10.4 with that of the plot in the center). If exchange of the fluorescent protein fragments between the interaction partners does not produce the predicted results, it is possible that the fluorescent protein fragments influence the specificity of the interaction. In this case, the relative efficiencies of complex formation cannot be determined using multicolor bimolecular fluorescence complementation analysis.

\section{Limitations of the Method}

The multicolor BiFC assay for measurement of the efficiencies of protein interactions in cells enables determination of the relative efficiencies of competition between alternative interaction partners for a shared partner, but this does not necessarily reflect their relative 


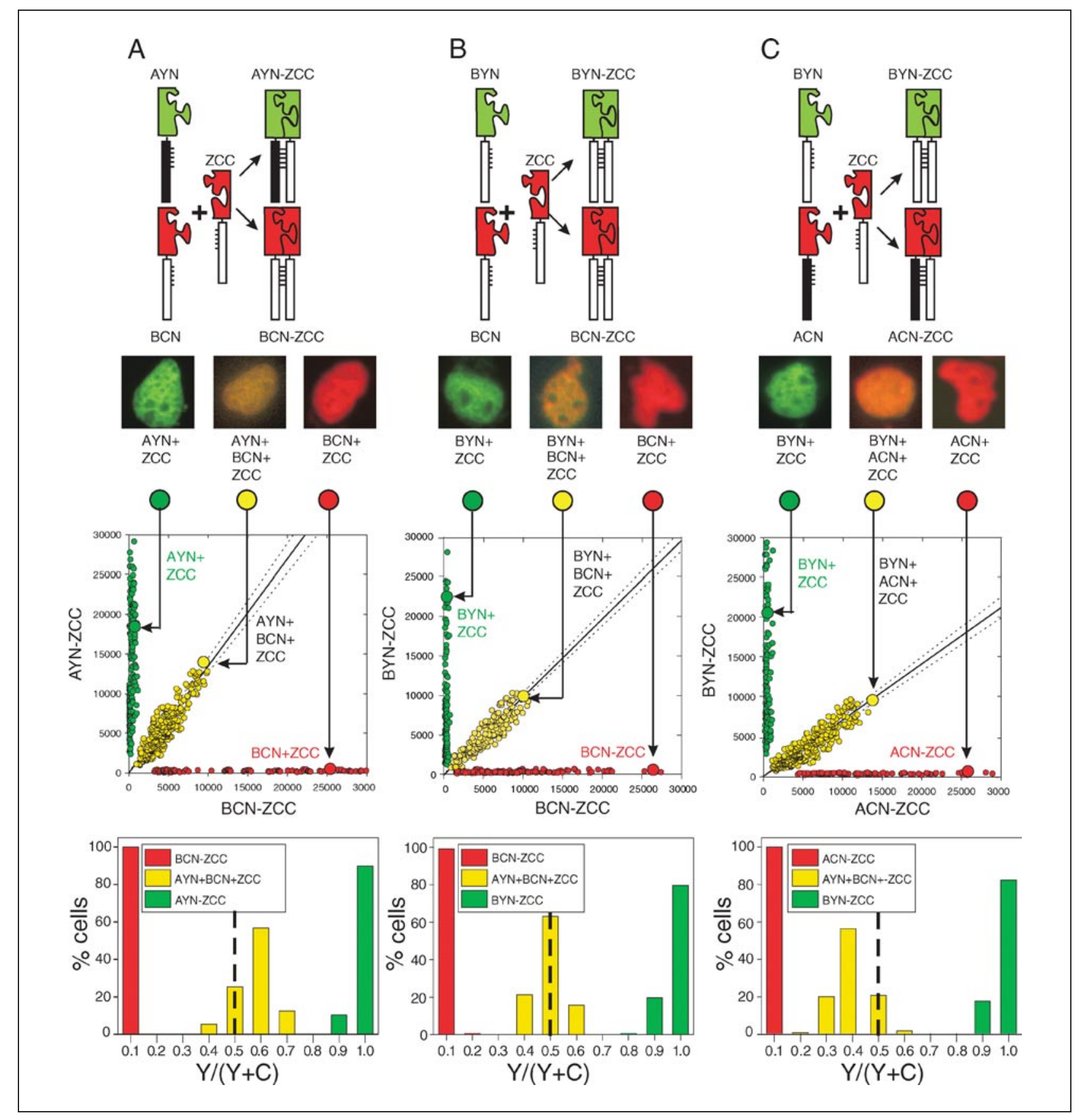

Figure 19.10.4 Legend at right.

Bimolecular

Fluorescence

Complementation

\subsubsection{2}


binding affinities, since the complexes are not at equilibrium for the entire time of the experiment. The association of the fluorescent protein fragments is relatively slow $\left(t_{1 / 2} \approx\right.$ $60 \mathrm{sec}$ ) and essentially irreversible in vitro (Hu et al., 2002). The relative efficiencies of complex formation reflect the competition between alternative interaction partners during the time before association of the fluorescent protein fragments. This is predicted to give a valid estimate of the relative efficiencies of complex formation by proteins with rapid exchange rates. However, interaction partners with very slow rates of association may not compete efficiently for complex formation in the assay.

The multicolor BiFC approach is based on comparison of the relative amounts of bimolecular fluorescent complexes with different spectral characteristics. Differences between the rates of degradation of proteins in different complexes could alter the steady-state ratio between the complexes and thereby affect the apparent efficiencies of complex formation. The relative fluorescence intensities should therefore be measured at several time points, and the steady-state levels of proteins should be compared by western analysis. The rates of degradation of the complexes can be compared by monitoring the decrease in fluorescence in cells treated with protein-synthesis inhibitors (e.g., cycloheximide). The rates of degradation of the fusion proteins can also be measured directly using pulse-chase analysis.

\section{Example of the Application of Quantitative Multicolor BiFC Analysis for Determination of the Relative Efficiencies of Complex Formation}

To examine the relative efficiencies of complex formation by protein $\mathrm{Z}$ with two alternative interaction partners, $\mathrm{A}$ and $\mathrm{B}$, the authors of this unit performed an experiment in which the proteins were fused to fluorescent protein fragments (A-YN, B-CN, and Z-CC) and were expressed separately and in combination (Grinberg et al., 2004). The fluorescence intensities of the alternative complexes (i.e., A-YN-Z-CC and B-CN-Z$\mathrm{CC})$ were measured when the complexes were expressed in separate cells and when they were expressed together in the same cell (Fig. 19.10.4A). As a reference, the fluorescent proteins were fused to the same interaction partners (B-YN, B-CN and Z-CC), and their fluorescence intensities were analyzed in parallel (Fig. 19.10.4B). The fluorescence intensities of the complexes were plotted in scatter plots (middle panels) and histograms

Figure 19.10.4 (at Left) Multicolor fluorescence complementation analysis of the competition for dimerization between alternative interaction partners. The relative efficiencies of complex formation between protein $\mathrm{Z}$ and two alternative interaction partners, $\mathrm{A}$ and $\mathrm{B}$, can be quantified by fusing them to fragments of different fluorescent proteins and expressing them in different combinations. (A) BiFC analysis of A-YN + Z-CC (green), B-CN + Z-CC (red) and A-YN + B-CN + Z-CC (yellow). (B) BiFC analysis of B-YN + Z-CC (green), B-CN + Z-CC (red) and B-YN + B-CN + Z-CC (yellow). (C) BiFC analysis of B-YN + Z-CC (green), A-CN + Z-CC (red) and B-YN + A$\mathrm{CN}+\mathrm{Z}-\mathrm{CC}$ (yellow). The diagrams at the top indicate the combinations of proteins expressed either pairwise or in three-way competition in each experiment. The images show cells expressing the proteins indicated below each image. The cells were imaged using filters (described in the instrumentation section) that distinguish the fluorescence emissions of $\mathrm{YN}-\mathrm{CC}$ (green) and $\mathrm{CN}-$ $\mathrm{CC}$ (red) complexes, and the images were superimposed. The fluorescence intensities of YN-CC complexes were plotted as a function of the fluorescence intensities of $\mathrm{CN}-\mathrm{CC}$ complexes in individual cells (middle row of graphs). The fluorescence intensities are shown in green and red for cells that express the interaction partners pairwise, and in yellow for cells that express two alternative interaction partners in direct competition. The best fit of a linear function to the data from cells coexpressing three proteins is shown. The slope of this function reflects the relative efficiencies of complex formation between the alternative interaction partners. The ratio between the fluorescence emissions from each complex $[\mathrm{Y} /(\mathrm{Y}+\mathrm{C})$ where $\mathrm{Y}$ represents $\mathrm{YN}-\mathrm{CC}$ and $\mathrm{C}$ represents $\mathrm{CN}-\mathrm{CC}$ ] was determined in individual cells in each population, and was plotted in histograms (lower row of graphs) using the same colors as in the middle row. This black-and-white facsimile of the figure is intended only as a placeholder; for a full-color version of the figure go to http://www.interscience.wiley.com/c_p/colorfigures.htm.

Identification of Protein Interactions

19.10.13

Supplement 41 
(lower panels) when the complexes were expressed separately (red and green) and when they were expressed together in the same cells (yellow). The ratio between the fluorescence intensities of the complexes reflects the relative efficiencies of complex formation between the alternative interaction partners.

When A-YN and B-CN were coexpressed with Z-CC, the fluorescence intensities produced by A-YN-Z-CC and B-CN-Z-CC exhibited a linear relationship with a slope of 1.3 and a $95 \%$ confidence interval of 0.08 (Fig. 19.10.4A, middle row). In comparison, when $\mathrm{B}-\mathrm{YN}$ and $\mathrm{B}-\mathrm{CN}$ were coexpressed with $\mathrm{Z}-\mathrm{CC}$, the fluorescence intensities produced by B-YN-Z-CC and B-CN-Z-CC exhibited a linear relationship with a slope of 0.98 and a 95\% confidence interval of 0.05 (Fig. 19.10.4B, middle row). Likewise, the distribution of ratios between the fluorescence intensities $[\mathrm{Y} /(\mathrm{Y}+\mathrm{C})]$ was shifted toward higher values for complexes formed by A-YN and B-CN with Z-CC compared to complexes formed by B-YN and B-CN with Z-CC (compare lower row of graphs for Fig. 19.10.4, panels $\mathrm{A}$ and $\mathrm{B})$. These results are consistent with the interpretation that $\mathrm{Z}$ favors complex formation with A over complex formation with $\mathrm{B}$. The absolute fluorescence intensities of A-YN-Z-CC and B-YN-Z-CC were comparable when expressed separately, suggesting that the efficiencies of association of the fluorescent protein fragments were comparable under conditions where no competitors for complex formation were present.

To confirm that the fluorescent protein fragments fused to the alternative interaction partners did not influence the relative efficiencies of complex formation, the authors exchanged the fragments between the interaction partners (i.e., B-YN, A-CN and $\mathrm{Z}$-CC) and compared the fluorescence intensities of bimolecular fluorescent complexes formed by these proteins (Fig. 19.10.4C). The fluorescence intensities of these complexes exhibited a linear relationship with a slope of 0.7 and a $95 \%$ confidence interval of 0.04 (Fig. 19.10.4C, middle row). Likewise, the distribution of fluorescence intensity ratios was shifted in the opposite directions compared to the proteins in which the fragments were fused to the opposite interaction partners (see lower row of graphs for Fig. 19.10.4; compare panel $\mathrm{C}$ with panels $\mathrm{A}$ and $\mathrm{B}$ ). These data are consistent with the interpretation that the difference between the efficiencies of bimolecular fluorescent complex formation by $\mathrm{A}$ and $\mathrm{B}$ fusions is not due to a difference between the fluorescent protein fragments fused to these proteins, but is caused by a difference between the efficiencies of complex formation between $\mathrm{A}$ and $\mathrm{Z}$ versus B and $\mathrm{Z}$ (Grinberg et al., 2004).

\section{Controls for Quantitative Multicolor BiFC Analysis}

The use of both absolute and relative competition methods for the quantification of relative efficiencies of complex formation is designed to correct for many of the factors that influence the fluorescence intensities of bimolecular fluorescent complexes formed by fragments of different fluorescent proteins. However, there are additional factors that need to be considered and examined as follows.

1. The relative levels of A-YN155 and B-CN155 expression affect the relative amounts of complexes formed. The alternative interaction partners must therefore be expressed at equal concentrations and the shared interaction partner must be expressed at a lower, limiting concentration. All protein concentrations should preferably be within the physiological range to observe the competition under normal cellular conditions. The levels of protein expression can be measured by immunoblot analysis using antibodies directed against the same epitope tag on the alternative interaction partners.

2. The efficiencies of association of different fluorescent protein fragments as well as the fluorescence intensities of the resulting bimolecular complexes can differ. It is therefore essential to determine the relative fluorescence intensities of the bimolecular fluorescent complexes when they are brought together by the same interaction 
partners (i.e., A-YN155, A-CN155, and Z-CC155). The relative fluorescence intensities of the two bimolecular fluorescent complexes (i.e., A-YN155-Z-CC155 and A-CN155-Z-CC155) reflect both differences between the efficiencies of association of the fluorescent protein fragments and differences between their fluorescence intensities when they are brought together by the same interaction partners.

3. The relative efficiencies of bimolecular fluorescence complementation can be affected by steric constraints imposed by the interaction partners on the association of the fluorescent protein fragments. To investigate this possibility, the fluorescent protein fragments can be fused to the interaction partners using several different extended linkers. If fusion proteins containing different linkers produce identical results, then the efficiencies of bimolecular complex formation are unlikely to be affected by steric constraints.

4. Different fluorescent protein fragments could have differential effects on the efficiencies of competition for the shared interaction partner. It is therefore essential to exchange the fluorescent protein fragments between the interaction partners to determine if differences between the fluorescent protein fragments affect the relative efficiencies of competition. This can also be verified in vitro by measuring the relative amounts of bimolecular fluorescent complexes formed when different ratios of the fluorescent protein fragments fused to the same interaction partners are mixed $(\mathrm{Hu}$ and Kerppola, 2003). If the amounts of complexes formed are proportional to the relative concentrations of fusion proteins in the reaction, then differences between the fluorescent protein fragments do not influence the relative efficiencies of complex formation.

\section{COMMENTARY}

\section{Background Information}

The biological functions of proteins are determined in large part by their interactions with other proteins in the physiological environment. Numerous protein interactions have been identified using genetic screens and in vitro binding assays. It is important to develop experimental approaches that allow determination of the subcellular locations of these interactions.

The BiFC assay has been used to visualize interactions between many structurally unrelated proteins (Hu et al., 2002; Atmakuri et al., 2003; Deppmann et al., 2003; von der Lehr et al., 2003; Yu et al., 2003; Bracha-Drori et al., 2004; de Virgilio et al., 2004; Farina et al., 2004; Grinberg et al., 2004; Hynes et al., 2004a,b; Kanno et al., 2004; Rackham and Brown, 2004; Rajaram and Kerppola, 2004; Remy and Michnick 2004a,b; Remy et al., 2004; Tsuchisaka and Theologis, 2004a,b; Tzfira et al., 2004; Walter et al., 2004; Wei et al., 2004; Zal and Gascoigne, 2004; Zhang et al., 2004). The authors of this unit and other investigators have determined the localization of complexes formed by Fos, Jun, and ATF-2 (Hu et al., 2002); c-Maf and Sox (Rajaram and Kerppola, 2004); BATF and Jun (Deppmann et al., 2003); p65, p50, and IkB
(Hu et al., 2002); Myc, Max, Mxi1, Mad3, and Mad4 (Grinberg et al., 2004); Skp2 and c-Myc (von der Lehr et al., 2003); and Brd2 and histone H4 (Kanno et al., 2004).

Protein interactions are crucial for the control of most cellular functions. Several methods have been developed to study protein interactions in living cells. One of the most commonly employed methods is FRET (UNIT 19.5). The FRET assay is based on the use of two fluorophores either chemically linked or genetically fused to two proteins whose interaction is to be examined. Compared to the $\mathrm{BiFC}$ assay, FRET analysis generally requires higher levels of protein expression to detect energy transfer. Also, structural information or a great deal of luck is required to place the two fluorophores within $100 \AA$ of each other, which is the maximal distance over which any significant energy transfer between fluorescent proteins can be detected. The fraction of proteins that form complexes must also be high to produce a sufficient change in the donor and acceptor fluorescence intensities. To exclude alternative interpretations of the results, numerous controls must be performed and the fluorescence intensities must be measured with high quantitative accuracy. Despite these limitations, FRET has been successfully used for
Identification of Protein Interactions

\subsubsection{5}

Supplement 41 
the analysis of many protein interactions in living cells (Sorkin et al., 2000; Li et al., 2001; Majoul et al., 2002; Hink et al., 2003; Larson et al., 2003; Miyawaki, 2003; Tsien, 2003). A great advantage of FRET over BiFC analysis is the reversibility of complex formation, which potentially allows analysis of the interaction under equilibrium conditions. FRET is therefore superior for studies of the kinetics of protein association and dissociation.

Bimolecular complementation between fragments of a variety of proteins has been used to investigate protein interactions in cells (Johnsson and Varshavsky, 1994; Rossi et al., 1997; Pelletier et al., 1998; Rossi et al., 1998; Ghosh, 2000; Galarneau et al., 2002; Wehrman et al., 2002; Paulmurugan and Gambhir, 2003). The basic principle is to fuse fragments of a reporter protein to the interaction partners. Selected fragments of many proteins are able to associate with each other to produce a functional complex. This complex can be detected by the use of chromogenic or fluorogenic substrates or ligands. Reporter proteins that have been used to date include ubiquitin (Johnsson and Varshavsky, 1994), $\beta$-galactosidase (Rossi et al., 1997), dihydrofolate reductase (Pelletier et al., 1998), luciferase (Paulmurugan and Gambhir, 2003), and $\beta$-lactamase (Galarneau et al., 2002; Wehrman et al., 2002). BiFC employs the classical principle of complementation between protein fragments (Ullmann et al., 1967, 1968). The advantage of the BiFC approach over other complementation approaches is that the reconstituted protein has strong intrinsic fluorescence that allows direct visualization of the protein complex. The complex can therefore be visualized with minimal perturbation of the cells. Using the BiFC approach, living cells can be observed over long periods, and the possibility of experimental manipulations altering the result is minimized. Potential problems with unequal distributions of the chromogenic or fluorogenic substrates or ligands are also avoided. Moreover, as described in Alternate Protocols 1 and 2, multiple protein interactions can be visualized in parallel using spectrally distinct bimolecular fluorescent complexes.

One limitation of the BiFC approach is the time required for fluorophore maturation. This prevents real-time detection of rapid changes in interactions using the BiFC assay. In addition, bimolecular fluorescent complex formation is irreversible in vitro. These characteristics prevent the use of the BiFC assay for studies of the dissociation and subunit exchange of protein complexes in cells.

\section{Characteristics of the BiFC assay}

The BiFC assay has several features that make it particularly useful for the study of protein interactions. First, it enables direct visualization of protein interactions and does not rely on their secondary effects. Second, the interactions can be visualized in living cells, eliminating potential artifacts associated with cell lysis or fixation. Third, the proteins are expressed in a relevant biological context, ideally at levels comparable to their endogenous counterparts. This increases the likelihood that they will reflect the properties of native proteins, including any post-translational modifications. Fourth, the BiFC assay does not require stoichiometric complex formation but can detect interactions between subpopulations of each protein. Fifth, multicolor BiFC analysis allows simultaneous visualization of multiple protein complexes in the same cell and enables analysis of the competition between alternative interaction partners for complex formation. Finally, BiFC does not require specialized equipment, apart from an inverted fluorescence microscope equipped with objectives that allow imaging of fluorescence in cells. The simple detection of bimolecular complex formation requires no post-acquisition image processing for interpretation of the data.

\section{Critical Parameters}

\section{Design of BiFC fusion constructs}

The fusion proteins for BiFC analysis must be designed with the specific proteins to be investigated as well as the purpose of the experiment in mind. Schematic diagrams of the different permutations of fusion proteins that can be used for BiFC analysis are shown in Figure 19.10.2. Some general guidelines for the design of fusion proteins for BiFC analysis are discussed below:

\section{Fragments of fluorescent proteins}

Several combinations of fluorescent protein fragments support bimolecular fluorescence complementation (Hu and Kerppola, 2003). The combinations of fluorescent protein fragments recommended for $\mathrm{BiFC}$ analysis are listed in Table 19.10.1. For most purposes, fragments of YFP truncated at residue 155 (designated YN155, containing N-terminal residues 1 to 154 , and $\mathrm{YC} 155$, containing of C-terminal residues 155 to 238) are recommended, as they exhibit a relatively high complementation efficiency when fused to many interaction partners, yet produce low fluorescence when fused to proteins that do not 
interact with each other. Fragments of YFP truncated at residue 173 (designated YN173 containing residues 1 to 172 , and $\mathrm{YC} 173$, containing residues 172 to 238) can also be used. Other combinations of fluorescent protein fragments can be also used ( $\mathrm{Hu}$ and Kerppola, 2003) as described in the multicolor BiFC assay (see Alternate Protocols 1 and 2).

\section{Positions of fusions}

The positions of the fusions must be determined empirically to fulfill three essential criteria:

1. The fusions must allow the fragments of the fluorescent proteins to associate with each other if the fusion proteins interact. Information about the structure and location of the interaction interface may be useful for this purpose. However, this information is not essential since BiFC vectors can be designed by screening multiple combinations of fusion proteins for fluorescence complementation. A simple strategy for the identification of fusion proteins that allow bimolecular fluorescence complementation is to fuse each of the fluorescent protein fragments to the $\mathrm{N}$ - and $\mathrm{C}$-terminal end of each interaction partner, and to test the fusion proteins for complementation in all eight combinations that contain both fragments of the fluorescent protein (Fig. 19.10.2).

2 . The fusions must not affect the localization or the stabilities of the proteins. This should be confirmed by comparing the localization and the level of expression of the fusion proteins with those of wild-type proteins lacking the fusions using indirect immunofluorescence and immunoblot (UNIT 10.10) analyses.

3. The fusions must not affect the functions of the proteins under investigation. Ideally, the protein functions should be validated using assays that evaluate all of the known functions of the endogenous proteins, and they should be performed under the conditions used to visualize the protein interactions.

\section{Linkers}

The linkers connect the fragments of the fluorescent proteins to the proteins of interest in the fusion proteins. The linkers must provide flexibility for independent motion of the fluorescent protein fragments and the interaction partners, allowing the fragments to associate when the proteins interact. The authors have used the RSIAT and RPACKIPNDLKQKVMNH linker sequences in many fusion constructs used for
BiFC analysis ( $\mathrm{Hu}$ et al., 2002; $\mathrm{Hu}$ and Kerppola, 2003). These linkers have been used for the visualization of interactions between many structurally unrelated proteins. The sequence AAANSSIDLISVPVDSR encoded by the multiple cloning sites of the PCMV-FLAG vector (Sigma) has also been successfully used as a linker in many BiFC experiments. Although these linker sequences worked well in the proteins examined, it is possible that the length or the sequence of the linker affects the complementation efficiency between other proteins.

\section{Design of constructs for multicolor BiFC analysis}

The basic principles for the design of $\mathrm{BiFC}$ constructs also apply to the design of multicolor BiFC constructs. The main difference is the use of fluorescent protein fragments that provide maximal spectral separation of the fluorescence signals from different bimolecular complexes. There are several combinations of fragments that can be used for multicolor BiFC analysis (Table 19.10.1). The use of CC155 (Cterminal fragment of CFP) paired with YN155 versus CN155 (N-terminal fragment of CFP) provides good spectral separation and high complementation efficiency. These combinations are therefore appropriate for the simultaneous analysis of two protein interactions. For the simultaneous analysis of more than two interactions, more selective interference filters and more complex spectral separation algorithms are required.

\section{Expression system}

The selection of an expression system should be based on the purpose of the experiment. To determine whether a pair of proteins can interact in cells and to determine the subcellular location of the complex, a transient overexpression system can be used. However, overexpression of proteins in cells can result in mislocalization of the proteins and formation of non-native complexes. To ensure that the observed fluorescence signal reflects native interactions, the fusion proteins should be expressed at levels comparable to the endogenous proteins. This can be done by the use of plasmids with weak promoters and plasmid vectors that do not replicate in mammalian cells. Also, cells can be transfected using small amounts of plasmid DNA, and they can be observed soon after transfection.

More consistent results can be obtained by using inducible expression vectors integrated into the genomes of stable cell lines (e.g.,
Identification of Protein Interactions 19.10.17 
pIND). This allows for the control of protein expression at relatively uniform levels in the entire cell population.

\section{Controls}

In order to determine if any fluorescence observed reflects a specific protein interaction, it is essential to include negative controls in each experiment. This is especially important because the fluorescent protein fragments are able to form fluorescent complexes with a low efficiency even in the absence of a specific interaction. This spontaneous complementation is generally reduced when the fragments are fused to proteins that do not interact with each other. The validity of bimolecular fluorescence complementation results must therefore be confirmed using fusions to proteins in which the interaction interface has been mutated $(\mathrm{Hu}$ et al., 2002; Grinberg et al., 2004). The mutant proteins must be fused to the fluorescent protein fragments in a manner identical to the wild-type protein. The mutation must not affect the stability or the subcellular location of the fusion protein. The level of expression and the localization of the mutant protein should be compared with the wild-type fusion protein by immunoblot (UNIT 10.10) and indirect immunofluorescence analyses. The efficiencies of fluorescence complementation by the wild-type and mutant proteins should be quantified and compared. If the interaction interface has not been previously characterized, it is possible to screen for mutations that alter the efficiency of bimolecular fluorescence complementation, and thereby to determine if the complementation reflects a specific interaction. The BiFC assay can therefore be used to determine whether two proteins interact in cells without prior knowledge of the location or the structural nature of the interaction interface.

\section{Protein concentrations}

The levels of expression of fusion proteins used for BiFC analysis can have a profound influence on the experimental results and on interpretation of the data. A high level of expression may produce non-native protein interactions, and may result in bimolecular complementation independent of specific protein interactions. It may also alter the subcellular distributions of the fusion proteins and alter cell functions. These may lead to the misinterpretation of the observed interactions. It is therefore important to control the levels of expression of the fusion proteins and to compare them with those of the endogenous proteins.
This can be accomplished by using regulated expression vectors and by making stably transfected cell lines. Differences between the levels of expression of the fusion proteins and their endogenous counterparts should also be considered when interpreting the results from competition studies using the multicolor BiFC assay.

\section{Time and temperature}

The time required for the detection of fluorescence varies depending on the complementation efficiency. For most productive interactions, transiently transfected cells exhibit fluorescence at 12 to $36 \mathrm{hr}$ after transfection. In the case of some interactions, fluorescence can be detected as early as $8 \mathrm{hr}$ after transfection (Hu et al., 2002; Grinberg et al., 2004). Long incubation times should be avoided since this may result in higher expression of fusion proteins and complementation due to nonspecific interaction. Since fluorophore maturation is sensitive to high temperature, incubation at $30^{\circ} \mathrm{C}$ for $30 \mathrm{~min}$ to several hours can increase the signal. Keep in mind that results obtained at $30^{\circ} \mathrm{C}$ should be carefully interpreted, as lowtemperature incubation may alter protein localization and/or interactions.

\section{Interpretation of lack of fluorescence complementation}

The lack of fluorescence complementation does not prove the absence of an interaction because fusion of nonfluorescent fragments to the proteins of interest can alter their structures, which could interfere with an interaction between the proteins. Moreover, the spatial arrangement of the fragments of fluorescent proteins may not allow bimolecular complex formation. Only in cases where fluorescence complementation can be induced by an extracellular signal can the lack of fluorescence complementation in the absence of the signal be tentatively interpreted to reflect the absence of an interaction or a change in complex architecture. If there is strong evidence to indicate that the proteins interact in cells, fluorescence complementation could be examined in different cell types or in the presence of different extracellular stimuli. Alternatively, additional fusion proteins containing different linker sequences could be tested.

\section{Quantification of the efficiency of bimolecular fluorescence complementation}

The efficiency of fluorescence complementation is defined as the fluorescence intensity produced by bimolecular fluorescent complex formation relative to the levels of fusion 
proteins present in the cell. The efficiencies of bimolecular fluorescence complementation by structurally unrelated proteins cannot be used to determine their efficiencies of complex formation, since many factors unrelated to the efficiency of complex formation influence the efficiency of bimolecular fluorescence complementation. However, in situations where all of these factors are expected to be identical, differences in the efficiency of bimolecular fluorescence complementation can provide information about the relative efficiencies of complex formation. Thus, the effects of single amino acid substitutions that do not alter the level of protein expression or its localization can be examined by quantifying the efficiencies of fluorescence complementation by the wild-type and mutated proteins (Hu et al., 2002; Hu and Kerppola, 2003). To quantify the efficiency of fluorescence complementation, it is necessary to include an internal control in the experiment to normalize for differences in the efficiencies of transfection and protein expression in individual cells. For this purpose, cells are cotransfected with plasmids encoding the two fusion proteins together with a plasmid encoding a full-length fluorescent protein with distinct spectral characteristics (e.g., CFP). The fluorescence intensities derived from both bimolecular fluorescence complementation (i.e., YFP) and the internal control (i.e., CFP) are measured in individual cells. The ratio of YFP to CFP emission is calculated after subtraction of background signal (Fig. 19.10.3). The ratio of YFP to CFP fluorescence is a measure of the efficiency of bimolecular fluorescence complementation. For structurally related proteins under carefully controlled conditions, the efficiency of complementation can provide information about the efficiency of the protein interaction.

\section{Anticipated Results}

If the fusion proteins under investigation support fluorescence complementation within 12 to $36 \mathrm{hr}$ after transfection, it is likely that the complementation reflects a specific interaction. This possibility must be verified by the analysis of mutated proteins. For proteins that display complementation after longer times, it is increasingly probable that the complementation results from a nonspecific association. If eight different combinations of aminoand carboxy-terminal fusions are tested and no complementation is observed, it is unlikely that additional combinations will produce fluorescence complementation, although this does not exclude the possibility that the proteins may interact.

\section{Time Considerations}

The construction of plasmid vectors for $\mathrm{BiFC}$ and multicolor BiFC analysis can be accomplished in 1 to 2 weeks. For transfection, preparation of the cells takes 1 day and transfection takes 1 hr using FuGENE 6. Next, 1 to 2 days incubation at $37^{\circ} \mathrm{C}$ is required. To promote chromophore maturation, incubation at $30^{\circ} \mathrm{C}$ for $30 \mathrm{~min}$ to several hours or overnight may be necessary. Imaging of the cells may take a few hours depending on how the data will be analyzed. Quantitative analysis requires about half a day to 1 day for each experiment. If everything goes smoothly, 3 to 4 weeks should be enough to obtain preliminary results. However, it takes much longer to validate the results by performing all the necessary controls. Confirmation of the interaction using cells stably transfected with inducible expression vectors also takes longer, since it requires the establishment of stable cell lines expressing the fusion proteins.

\section{Literature Cited}

Atmakuri, K., Ding, Z., and Christie, P.J. 2003. VirE2, a type IV secretion substrate, interacts with the VirD4 transfer protein at cell poles of Agrobacterium tumefaciens. Mol. Microbiol. 49:1699-1713.

Bracha-Drori, K., Shichrur, K., Katz, A., Oliva, M., Angelovici, R., Yalovsky, S., and Ohad, N. 2004. Detection of protein-protein interactions in plants using bimolecular fluorescence complementation. Plant J. 40:419-427.

Deppmann, C.D., Thornton, T.M., Utama, F.E., and Taparowsky, E.J. 2003. Phosphorylation of BATF regulates DNA binding: A novel mechanism for AP-1 (activator protein-1) regulation. Biochem. J. 374:423-431.

de Virgilio, M., Kiosses, W.B., and Shattil, S.J. 2004. Proximal, selective, and dynamic interactions between integrin alpha II beta 3 and protein tyrosine kinases in living cells. J. Cell Biol. 165:305-311.

Farina, A., Hattori, M., Qin, J., Nakatani, Y., Minato, N., and Ozato, K. 2004. Bromodomain protein Brd4 binds to GTPase-activating SPA-1, modulating its activity and subcellular localization. Mol. Cell. Biol. 24:9059-9069.

Galarneau, A., Primeau, M., Trudeau, L.E., and Michnick, S.W. 2002. Beta-lactamase protein fragment complementation assays as in vivo and in vitro sensors of protein protein interactions. Nat. Biotechnol. 20:619-622.

Ghosh, I., Hamilton, A.D., and Regan, L. 2000. Antiparallel leucine zipper-directed protein reassembly: Application to the green fluorescent protein. J. Am. Chem. Soc. 122:5658-5659.
Identification

of Protein

Interactions

19.10.19 
Grinberg, A.V., Hu, C.D., and Kerppola, T.K. 2004. Visualization of Myc/Max/Mad family dimers and the competition for dimerization in living cells. Mol. Cell. Biol. 24:4294-4308.

Hink, M.A., Borst, J.W., and Visser, A.J. 2003. Fluorescence correlation spectroscopy of GFP fusion proteins in living plant cells. Methods Enzymol. 361:93-112.

Hu, C.D. and Kerppola, T.K. 2003. Simultaneous visualization of multiple protein interactions in living cells using multicolor fluorescence complementation analysis. Nat. Biotechnol. 21:539545.

Hu, C.D., Chinenov, Y., and Kerppola, T.K. 2002. Visualization of interactions among bZIP and Rel family proteins in living cells using bimolecular fluorescence complementation. Mol. Cell 9:789-798.

Hynes, T.R., Mervine, S.M., Yost, E.A., Sabo, J.L., and Berlot, C.H. 2004a. Live cell imaging of G(s) and the beta(2)-adrenergic receptor demonstrates that both alpha(s) and beta(1)gamma(7) internalize upon stimulation and exhibit similar trafficking patterns that differ from that of the beta(2)-adrenergic receptor. J. Biol. Chem. 279:44101-44112.

Hynes, T.R., Tang, L.N., Mervine, S.M., Sabo, J.L., Yost, E.A., Devreotes, P.N., and Berlot, C.H. $2004 \mathrm{~b}$. Visualization of $\mathrm{G}$ protein beta gamma dimers using bimolecular fluorescence complementation demonstrates roles for both beta and gamma in subcellular targeting. J. Biol. Chem. 279:30279-30286.

Johnsson, N. and Varshavsky, A. 1994. Split ubiquitin as a sensor of protein interactions in vivo. Proc. Natl. Acad. Sci. U.S.A. 91:10340-10344.

Kanno, T., Kanno, Y., Siegel, R.M., Jang, M.K., Lenardo, M.J., and Ozato, K. 2004. Selective recognition of acetylated histones by bromodomain proteins visualized in living cells. Mol. Cell 13:33-43.

Larson, D.R., Ma, Y.M., Vogt, V.M., and Webb, W.W. 2003. Direct measurement of Gag-Gag interaction during retrovirus assembly with FRET and fluorescence correlation spectroscopy. J. Cell Biol. 162:1233-1244.

Li, H.Y., Ng, E.K., Lee, S.M., Kotaka, M., Tsui, S.K., Lee, C.Y., Fung, K.P., and Waye, M.M. 2001. Protein-protein interaction of FHL3 with FHL2 and visualization of their interaction by green fluorescent proteins (GFP) two-fusion fluorescence resonance energy transfer (FRET). J. Cell. Biochem. 80:293-303.

Majoul, I., Straub, M., Duden, R., Hell, S.W., and Soling, H.D. 2002. Fluorescence resonance energy transfer analysis of protein-protein interactions in single living cells by multifocal multiphoton microscopy. J. Biotechnol. 82:267-277.

Miyawaki, A. 2003. Visualization of the spatial and temporal dynamics of intracellular signaling. Dev. Cell 4:295-305.

Nagai, T., Sawano, A., Park, E.S., and Miyawaki, A. 2001. Circularly permuted green fluorescent proteins engineered to sense $\mathrm{Ca}^{2+}$. Proc. Natl. Acad. Sci. U.S.A. 98:3197-202.

Paulmurugan, R. and Gambhir, S.S. 2003. Monitoring protein-protein interactions using split synthetic renilla luciferase protein-fragmentassisted complementation. Anal. Chem. 75:1584-1589.

Pelletier, J.N., Campbell-Valois, F.X., and Michnick, S.W. 1998. Oligomerization domaindirected reassembly of active dihydrofolate reductase from rationally designed fragments. Proc. Natl. Acad. Sci. U.S.A. 95:12141-12146.

Rackham, O. and Brown, C.M. 2004. Visualization of RNA-protein interactions in living cells: FMRP and IMP1 interact on mRNAs. EMBO J. 23:3346-3355.

Rajaram, N. and Kerppola, T. 2004. Transcription activation by Maf and Sox, and their subnuclear localization are disrupted by a mutation in Maf that causes cataract. Mol. Cell. Biol. 24:56945709.

Remy, I. and Michnick, S.W. 2004a. A cDNA library functional screening strategy based on fluorescent protein complementation assays to identify novel components of signaling pathways. Methods 32:381-388.

Remy, I. and Michnick, S.W. 2004b. Regulation of apoptosis by the $\mathrm{Ft} 1$ protein, a new modulator of protein kinase B/Akt. Mol. Cell. Biol. 24:14931504.

Remy, I., Montmarquette, A., and Michnick, S.W. 2004. PKB/Akt modulates TGF-beta signalling through a direct interaction with Smad3. Nature Cell Biol. 6:358-365.

Rossi, F., Charlton, C.A., and Blau, H.M. 1997. Monitoring protein-protein interactions in intact eukaryotic cells by beta-galactosidase complementation. Proc. Natl. Acad. Sci. U.S.A. 94:8405-8410.

Rossi, F.M., Guicherit, O.M., Spicher, A., Kringstein, A.M., Fatyol, K., Blakely, B.T., and Blau, H.M. 1998. Tetracycline-regulatable factors with distinct dimerization domains allow reversible growth inhibition by p16. Nat. Genet. 20:389-393.

Sorkin, A., McClure, M., Huang, F., and Carter, R. 2000. Interaction of EGF receptor and grb2 in living cells visualized by fluorescence resonance energy transfer (FRET) microscopy. Curr. Biol. 10:1395-1398.

Tsien, R.Y. 2003. Imagining imaging's future. Nat. Rev. Mol. Cell. Biol. Suppl. SS16-21.

Tsuchisaka, A. and Theologis, A. 2004a. Heterodimeric interactions among the 1amino-cyclopropane-1-carboxylate synthase polypeptides encoded by the Arabidopsis gene family. Proc. Natl. Acad. Sci. U.S.A. 101:22752280.

Tsuchisaka, A. and Theologis, A. 2004b. Unique and overlapping expression patterns among the arabidopsis 1-amino-cyclopropane-1carboxylate synthase gene family members. Plant Physiol. 136:2982-3000. 
Tzfira, T., Vaidya, M., and Citovsky, V. 2004. Involvement of targeted proteolysis in plant genetic transformation by Agrobacterium. Nature 431:87-92.

Ullmann, A., Jacob, F., and Monod, J. 1967. Characterization by in vitro complementation of a peptide corresponding to an operator-proximal segment of the beta-galactosidase structural gene of Escherichia coli. J. Mol. Biol. 24:339343.

Ullmann, A., Jacob, F., and Monod, J. 1968. On the subunit structure of wild-type versus complemented beta-galactosidase of Escherichia coli. J. Mol. Biol. 32:1-13.

von der Lehr, N., Johansson, S., Wu, S., Bahram, F., Castell, A., Cetinkaya, C., Hydbring, P., Weidung, I., Nakayama, K., Nakayama, K.I., Soderberg, O., Kerppola, T.K., and Larsson, L.G. 2003. The F-box protein Skp2 participates in c-Myc proteosomal degradation and acts as a cofactor for c-Myc-regulated transcription. Mol. Cell 11:1189-1200.

Walter, M., Chaban, C., Schutze, K., Batistic, O., Weckermann, K., Nake, C., Blazevic, D., Grefen, C., Schumacher, K., Oecking, C., Harter, K., and Kudla, J. 2004. Visualization of protein interactions in living plant cells using bimolecular fluorescence complementation. Plant J. 40:428-438.
Wehrman, T., Kleaveland, B., Her, J.H., Balint, R.F., and Blau, H.M. 2002. Protein-protein interactions monitored in mammalian cells via complementation of beta-lactamase enzyme fragments. Proc. Natl. Acad. Sci. U.S.A. 99:3469-3474.

Wei, G.H., Liu, D.P., and Liang, C.C. 2004. Charting gene regulatory networks: Strategies, challenges and perspectives. Biochem. J. 381:1-12.

Yu, H., West, M., Keon, B.H., Bilter, G.K., Owens, S., Lamerdin, J., and Westwick, J.K. 2003. Measuring drug action in the cellular context using protein-fragment complementation assays. Assay Drug Dev.Technol. 1:811-822.

Zal, T. and Gascoigne, N.R. 2004. Using live FRET imaging to reveal early protein-protein interactions during $\mathrm{T}$ cell activation. Curr. Opin. Immunol. 16:418-427.

Zhang, S.F., Ma, C., and Chalfie, M. 2004. Combinatorial marking of cells and organelles with reconstituted fluorescent proteins. Cell 119:137144.

Contributed by Chang-Deng $\mathrm{Hu}$, Asya V. Grinberg, and Tom K. Kerppola

Howard Hughes Medical Institute and University of Michigan Medical School Ann Arbor, Michigan
Identification

of Protein

Interactions

19.10.21 